\title{
Paraganglioma de mediastino com metástases pulmonares *
}

\author{
Pulmonary metastasis of mediastinal paraganglioma
}

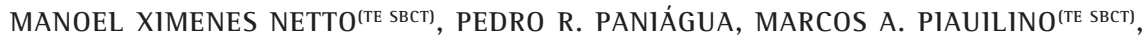

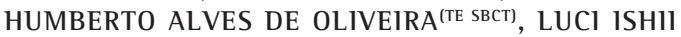

Descrevemos uma paciente de 27 anos que se apresentou com paraganglioma de mediastino anterior e médio e nódulos pulmonares bilaterais. 0 tratamento consistiu na ressecção das lesões pulmonares através de toracotomia anterior bilateral transesternal e retirada do paraganglioma com auxílio de circulação extracorpórea. Como tratamento neoadjuvante foram usadas radioterapia e quimioterapia. A evolução pósoperatória foi satisfatória, e catorze meses depois a paciente encontrava-se assintomática.

J Bras Pneumol 2005; 31(1): 76-9.
Herein, we describe the case of a 27-year-old female presenting with paraganglioma of the anterior and middle mediastinum and bilateral pulmonary nodules. Treatment consisted of pulmonary resection by anterior bilateral thoracotomy and transverse sternotomy, in which the paraganglioma was excised with the aid of extracorporeal circulation. As neoadjuvant treatments, radiotherapy and chemotherapy were applied. Postoperative evolution was uneventful, and the patient was classified as asymptomatic after 14 months.
Descritores : Tumor de mediastino. Paraganglioma. Metástases pulmonares.
Key words: Mediastinal tumor. Paraganglioma. Lung metastasis

\section{INTRODUÇÃO}

Feocromocitomas são tumores derivados das células cromafínicas, altamente vascularizados e categorizados como os tumores dos $10 \%$, porque 10\% são bilaterais, 10\% são malignos ou múltiplos, $10 \%$ são familiares e 10\% se apresentam em localização extra-adrenal, e por isso são chamados paragangliomas. 0 paraganglioma foi descrito pela primeira vez em 1.950 por Lattes ${ }^{(1)}$. Tanto os tumores funcionantes como os não funcionantes podem originar-se nas proximidades de qualquer área da cadeia neural simpática, e menos de $2 \%$ dos tumores secretores de catecolaminas são originários do tórax. Pouco mais de 150 casos de paraglangliomas intratorácicos foram descritos na literatura, e dois terços deles eram localizados no mediastino anterior e médio ${ }^{(2,3)}$.

Paragangliomas torácicos são tumores raros, altamente vascularizados, de origem neuroendócrina e que são tratados pela ressecção cirúrgica quando possível. São neoplasias de crescimento lento, localmente invasivas e com alto indice de recidiva. A sobrevida global é de $62 \%$, mas apenas $36,7 \%$ permanecem livres da doença, 
com um tempo de sobrevida de $98,2 \pm 11,7$ meses. A sobrevida nos casos de ressecção completa é de $84,6 \%$, e de $50 \%$ nos casos de ressecção parcial ou biópsia ${ }^{(2)}$. Esses tumores podem ser divididos em quatro tipos de acordo com a sua localização: branquiométrico, associado com os vasos arteriais e nervos cranianos; intravagal; aórtico simpático; e visceral autônomo.

Os paraglangliomas podem ser multicêntricos em 43\% dos casos e não parece haver uma distribuição específica ${ }^{(3)}$. Em 14\% dos casos a tríade de Carney tem sido descrita, a qual consiste numa síndrome não familiar, acometendo mulheres jovens que apresentam leiomiossarcoma epitelioide gástrico e condromas pulmonares, além de paraganglioma extra-adrenal funcionante ${ }^{(4)}$.

0 presente trabalho tem como finalidade apresentar o caso de uma paciente com paraganglioma de mediastino e metástases pulmonares, cuja opção cirúrgica foi a ressecção dos nódulos pulmonares, de forma bilateral e simultânea, e exérese do paraganglioma com auxílio de circulação extracorpórea.

\section{RELATO DO CASO}

Uma paciente de 27 anos foi encaminhada para avaliação de tumor em mediastino anterior e médio e nódulo pulmonar em lobo inferior direito e lobo superior esquerdo (Figura 1).

Foi submetida à biópsia através de incisão paraesternal com abertura do pericárdio. 0 diagnóstico estabelecido foi de paraganglioma, com positividade para sinaptofisina, cromogranina e proteína S 100, e negativo para panceratinina. A pesquisa cintilográfica com metaiodobenzilguanidina foi negativa. A pressão arterial era normal. 0 estudo de imagem pela angiorressonâcia de tórax revelou os detalhes do paraganglioma, posicionado entre o arco aórtico, a artéria pulmonar e o átrio direito (Figura 2).

A paciente foi submetida à ressecção das metástases pulmonares através de toracotomia anterior bilateral transesternal, seguida do estabelecimento de circulação extracorpórea e ressecção do paraganglioma, posicionado entre a aorta, tronco da artéria pulmonar e átrio direito, acometendo a adventícia dos vasos e pericárdio (Figura 3).

A histologia e imunohistoquímica confirmaram o diagnóstico de paraganglioma, inclusive nas lesões pulmonares e pericárdio (Figura 4).

Foram colocados clipes metálicos no local da ressecção. No pós-operatório a paciente foi submetida à radioterapia nos locais de ressecção, orientada pelos clipes, e também à quimioterapia sistêmica, com o uso de ciclofosfamida, vincristina, doxorubicina e dacarbazina. A paciente teve excelente evolução pós-operatória, e obteve alta hospitalar no quinto dia. Quinze meses depois, encontrava-se assintomática.

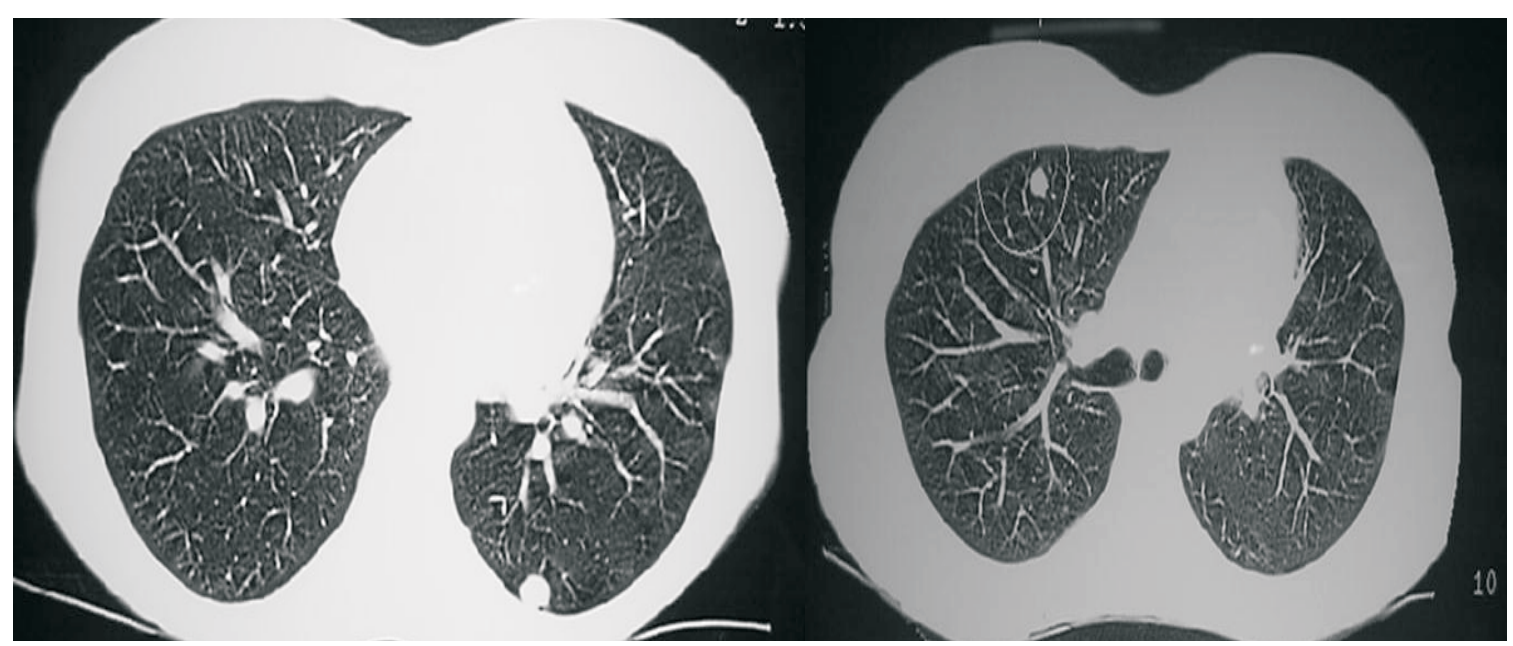

Figura 1 - Tomografia computadorizada mostrando nódulos pulmonares localizados no lobo inferior esquerdo e superior direito 


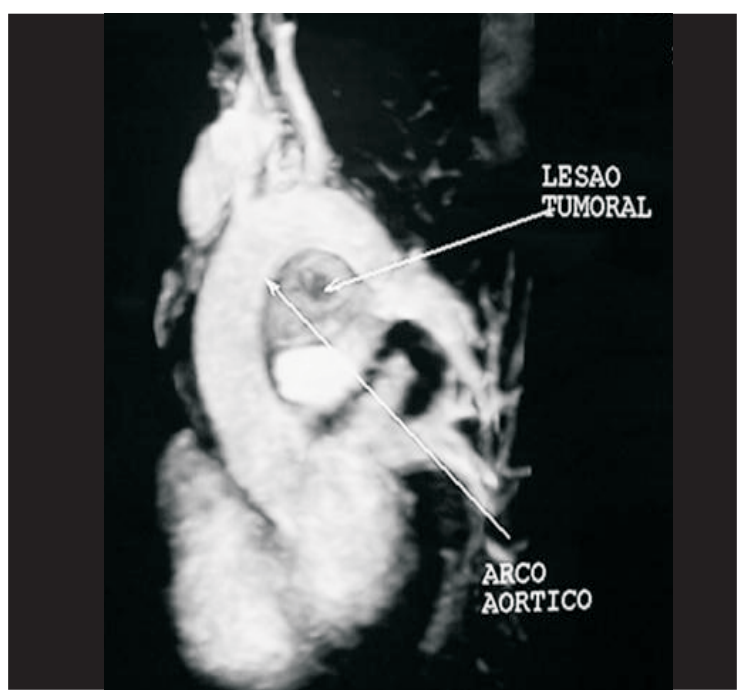

Figura 2 - Angiorressonância magnética mostrando o paraganglioma

\section{DISCUSSÃO}

Entre as diversas modalidades de imagem utilizadas para localizar os paragangliomas intratorácicos, a ressonância magnética, especialmente a angiorressonancia, é o método mais eficiente $(100 \%)$ e o escaneamento pelo metaiodobenzilguanidina pode ser bastante eficaz, desde que usado scanner de alta resolução. Os resultados falso negativos foram encontrados nas seguintes proporções num estudo de 236 pacientes portadores de paraganglioma da Clínica Mayo: 0\%

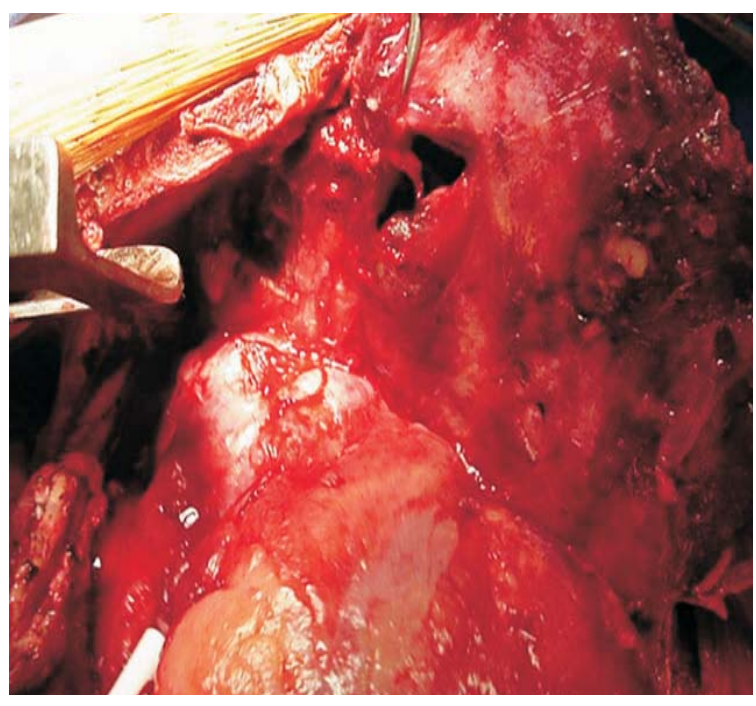

Figura 3 - Paraganglioma localizado na altura do arco aórtico

para a ressonância magnética, 5,8\% para a tomografia computadorizada, 3,4\% para a angiografia, 10,7\% para a ultra-sonografia e 39\% para a cintilografia com o metaiodobenzilguanidina ${ }^{(5)}$.

A maioria desses tumores, quando localizados no mediastino posterior, apresentam pouca dificuldade técnica na abordagem cirúrgica, ao contrário do que acontece com aqueles localizados no mediastino anterior e médio. Num total de 104 pacientes submetidos à ressecção de paragangliomas de mediastino, 3,8\% necessitaram do auxílio de

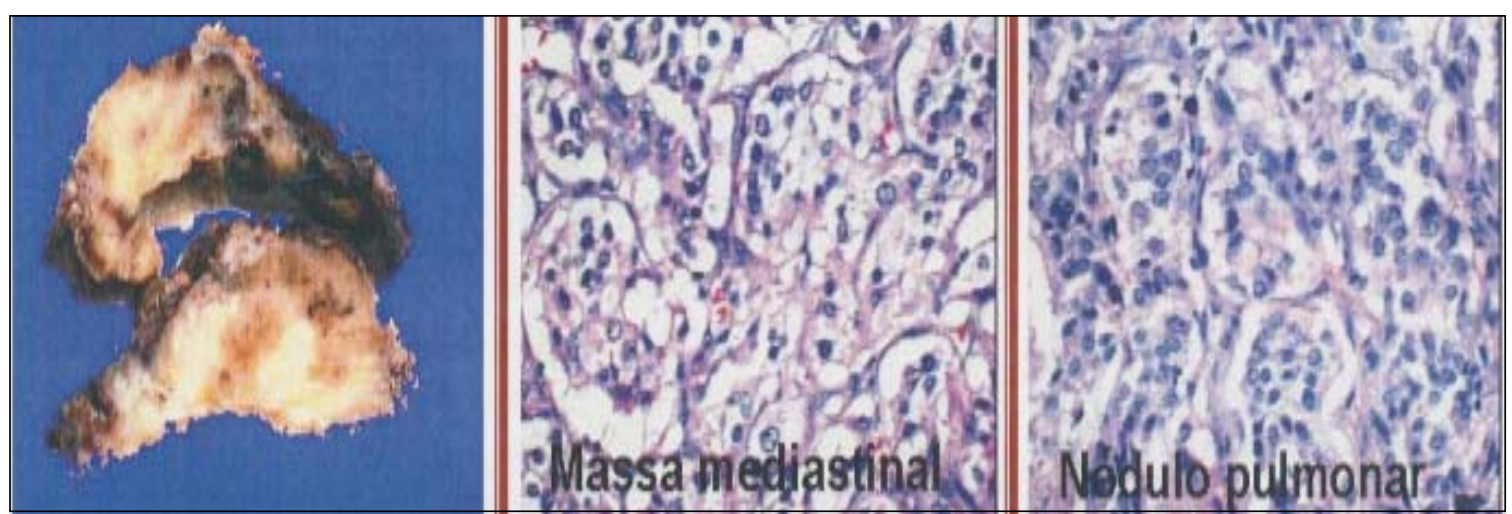

Figura 4 - A: aspecto macroscópico; B: histologia do paraganglioma evidenciando células poligonais dispostas em estroma fibroso, ricamente vascularizado e citoplasma granuloso; C: nódulo pulmonar com as mesmas características do tumor original. 
circulação extracorpórea a fim de se efetivar a remoção da massa tumoral com segurança ${ }^{(2-6)}$.

A radioterapia tem sido considerada como parcialmente eficiente no tratamento do paraganglioma. Ela pode diminuir o crescimento ou induzir a regressão parcial, mas a cura não deve ser esperada. Em nossa paciente optamos por complementar a cirurgia com irradiação do leito tumoral, além da quimioterapia, pois achamos que, apesar da radicalidade dos procedimentos cirúrgicos, não houve ressecção completa da massa tumoral. 0 regime quimioterápico usado em nossa paciente consistiu em ciclofosfamida, vincristina, doxorubicina e dacarbazina ${ }^{(7)}$.

Em virtude da extrema vascularidade desses tumores, em determinadas ocasiões a embolização prévia pode se tornar necessária, conforme descrito por Rakovich et al. ${ }^{(8)}$.

Em conclusão, os paragangliomas são tumores raros, de patologia complexa, que requerem tratamento multidisciplinar e, em centros capacitados, o uso de todos os recursos disponíveis no seu manuseio.

\section{AGRADECIMENTOS}

Aos professores Carlos E. Bachi, patologista da Faculdade de Medicina da Universidade Estadual
Paulista, e Saul Sester, Chefe da Divisão de Patologia Cirúrgica da Ohio State University, School of Medicine, Columbus, $\mathrm{OH}$, EUA.

\section{REFERÊNCIAS}

1. Lattes R. Non chromaffin paraganglioma of ganglion nodosum, carotid body and aortic arch bodies. Cancer 1950; 3: 667-94

2. Lamy AL, Fradet GJ, Luoma A, Nelems B. Anterior and middle mediastinum paraganglioma : Complete resection is the treatment of choice. Ann Thorac Surg 1994; 57: 249-52

3. Herrera MF, van Heerden JA, Puga FJ. Mediastinal paraganglioma: a surgical experience. Ann Thorac Surg 1993; 56: 1096-1100

4. Carney JA. The triad of gastric epitheloid leiomyosarcoma, pulmonary condroma and functioning extra-adrenal paraganglioma: a five year review. Medicine 1983; 62: 159-9

5. Erikson D, Kudva Y, Ebersold M. Benign paragangliomas: Clinical presentation and treatment outcomes in 236 patients. J Clin Endocrinol Metabol 2001; 86:5210-6

6. Andrade CF, Camargo S, Zanchet M, Felicetti JC, Cardoso PF. Nonfunctioning paraganglioma of the aortopulmonary window. Ann Thorac Surg 2003;75:1950-1

7. Patel SR, Winchester DJ, Benjamin RS. A 15 year experience with chemotherapy of patients with paraganglioma. Cancer 1995; 76: 1476-80

8. Rakovich G, Ferraro P, Therasse E, Duranceau A. Preoperative embolization in the management of a mediastinal paraganglioma. Ann Thorac Surg 2001; 72: 601-3 